\title{
Efeitos mecânicos causados pela variação da inclinação do parafuso excêntrico no orifício da placa de autocompressão*
}

\author{
Mechanical effects caused by the variation of the load screw \\ inclination in the orifice of the dynamic compression plate
}

\author{
Rafael Lara de Freitas ${ }^{1}$, Cleber Antonio Jansen Paccola², \\ ANTÔNIO CARlos SHIMANO ${ }^{3}$, MaURícIo KFURI JÚNIOR ${ }^{4}$
}

\section{RESUMO}

Objetivo: Avaliar os efeitos mecânicos da inclinação do parafuso excêntrico sobre a compressão axial com placa. Métodos: Corpos de prova artificiais simulando fragmentos de osso diafisário foram fixados com placa $D C P^{\circledR}$ de 4,5mm de sete orifícios. Uma célula de carga registrou as forças de compressão axial no intervalo entre os fragmentos. Guias de perfuração excêntrica com inclinações em relação ao plano longitudinal e transversal da placa foram confeccionados para o experimento. A compressão foi medida em dois diferentes sítios do foco de fratura virtual. De acordo com a magnitude da inclinação e sua direção em relação ao orifício da placa, oito diferentes grupos foram constituídos. Os ensaios mecânicos registraram a força máxima e a força máxima efetiva. Resultados: A inclinação do parafuso em direção con-

* Trabalho realizado no Laboratório de Bioengenharia da Faculdade de Medicina de Ribeirão Preto da Universidade de São Paulo - USP - Ribeirão Preto (SP), Brasil, para obtenção de título de Mestre em Medicina sob orientação do Prof. Dr. Mauricio Kfuri Junior.

Trabalho realizado com recursos próprios e sem conflito de interesses.

1. Mestre em Medicina pela Faculdade de Medicina de Ribeirão Preto da Universidade de São Paulo - USP - Ribeirão Preto (SP), Brasil. trária à da rampa de deslizamento do orifício da placa foi acompanhada de diminuição nos valores médios de força máxima e força máxima efetiva de compressão $0^{\circ}>10^{\circ} \mathrm{i}(\mathrm{p}<0,001), 0^{\circ}>20^{\circ} \mathrm{i}(\mathrm{p}<0,001), 0^{\circ}$ $>25^{\circ} \mathrm{i}(\mathrm{p}<0,001)$. Conclusão: Em modelo experimental, utilizando-se placas do tipo $D C P^{\circledR}$ não pré-tensionadas, a inclinação na inserção de parafusos excêntricos promoveu alterações nos valores médios da força de compressão axial. Houve diferenças significativas $(p<0,05)$ para menos na compressão axial obtida na cortical subjacente à placa, quando da inserção de parafusos inclinados no plano longitudinal com direção oposta à da rampa de deslizamento do orifício $D C P^{\circledR}$.

Descritores - Fixação interna de fraturas/métodos; Placas ósseas; Parafusos ósseos; Biomecânica

2. Professor Titular do Departamento de Biomecânica, Medicina e Reabilitação do Aparelho Locomotor da Faculdade de Medicina de Ribeirão Preto da Universidade de São Paulo - USP - Ribeirão Preto (SP), Brasil.

3. Doutor, Engenheiro Mecânico, Professor do Departamento de Biomecânica, Medicina e Reabilitação do Aparelho Locomotor da Faculdade de Medicina de Ribeirão Preto da Universidade de São Paulo - USP - Ribeirão Preto (SP), Brasil.

4. Doutor, Professor do Departamento de Biomecânica, Medicina e Reabilitação do Aparelho Locomotor da Faculdade de Medicina de Ribeirão Preto da Universidade de São Paulo - USP - Ribeirão Preto (SP), Brasil.

Endereço para correspondência: Rafael Lara de Freitas, Av. Portugal 2.800, apto, 1.053, Bairro City Ribeirão - 14020-380 - Ribeirão Preto (SP), Brasil. E-mail: dr.rafalara@ig.com.br

Recebido em 30/10/07. Aprovado para publicação em 18/3/08.

Copyright RBO2008 


\section{ABSTRACT}

Objective: To evaluate the mechanical effects of sloping the load screw on the axial compression with a dynamic plate. Methods: Artificial parts simulating shaft fragments were fixated with a $4.5 \mathrm{~mm}, 7$ orifice $D C P^{\circledR}$ plate. A load cell recorded axial compression loads in the space between fragments. Eccentric perforation guides with inclinations to the longitudinal and transversal plan of the plate were made for the experiment. Compression was measured in two different sites of the virtual fracture focus. Eight different groups were formed according to the magnitude of inclination and its direction based on the plate orifice. Mechanical assays recorded maximum load and effective maximum load. Results: Screw inclination contrary to the plate orifice sliding slope was related to decreased mean values of maximum compression load and maximum effective compression load $0^{\circ}>10^{\circ} i(p<0,001), 0>20^{\circ} i(p<0,001), 0^{\circ}>$ $25^{\circ} i(p<0,001)$. Conclusion: In an experimental model, using non pre-stressed $D C{ }^{\circledR}$ plates, the inclination upon inserting load screws brought about changes in the mean values of axial compression load. There significant differences $(p<0,05)$ to lower axial compression obtained in the cortical adjacent to the plate when the screws were inserted in inclination with the longitudinal plane in the opposite direction of the sliding slope of the $D C P^{\circledR}$ orifice.

Keywords - Fracture fixation, internal/methods; Bone plates; Bone screws; Biomechanics

\section{INTRODUÇÃO}

Baseado nos trabalhos de Danis, o Grupo AO protagonizou os princípios básicos de fixação interna, então fundamentados na redução anatômica, fixação interna estável, preservação do suprimento sanguíneo e mobilização ativa indolor ${ }^{(1-2)}$. A busca de osteossínteses rígidas conduziu ao desenvolvimento de uma nova placa de autocompressão: Dynamic Compression Plate $\left(D C P^{\circledR}\right)^{(3)}$. O fundamento mecânico para obtenção da compressão axial com essas placas baseou-se no fato de que a congruência entre a secção da cabeça esférica de um parafuso e a secção do orifício da placa cilíndri- ca promoveria estabilidade lateral do parafuso no orifício, condição não obtida, até então, pelos sistemas de placa de compressão existentes. A inserção excêntrica do parafuso no orifício da placa de autocompressão permite o deslizamento do fragmento ósseo subjacente à placa, levando à compressão interfragmentária axial. A congruência entre placa e cabeça do parafuso ocorreria não apenas na sua posição de ajuste final, mas também durante o processo de aperto do parafuso. $\mathrm{O}$ sistema de rampa inclinada do orifício, sem quinas ortogonais, permitiria diminuir a deformação indesejável dos parafusos no ato da inserção, aumentando a força de compressão. A geometria esférica da cabeça dos parafusos permitiria ainda a sua inclinação, tanto no plano longitudinal quanto transversal.

No início da década de 90 ficou caracterizada a transição de um enfoque puramente mecânico para uma abordagem mais biológica no tratamento das fraturas ${ }^{(4-5)}$.

O desenvolvimento da nova unidade de acoplamento com superfície rosqueada entre orifício da placa e cabeça de parafuso inaugurou uma nova fase no tratamento cirúrgico das fraturas. Introduzia-se, assim, o conceito de implantes que atuavam como fixadores externos, mas aplicados bem próximo ao osso: os fi$x^{x a d o r e s}$ internos $^{(6-8)}$. Os fixadores internos, assim como os externos, asseguravam algum grau de movimento no foco de fratura, agindo pelo princípio da estabilidade relativa e levando à consolidação mediante a formação de calo ósseo ${ }^{(9)}$.

Em 2003, surgiu a Locking Compression Plate $\left(L C P^{\circledR}\right)$ com o objetivo de oferecer em um único sistema todas as opções possíveis de fixação do osso por uma placa. O elemento novo do sistema era mais uma vez o orifício do parafuso, denominado sistema combinado ${ }^{(9-10)}$. Esse orifício, em formato de um oito, era constituído de duas metades. Uma metade idêntica à do orifício $D C P^{\circledR}$, destinada ao uso de parafusos convencionais, e a outra, dotada de ranhuras para a utilização de parafusos com cabeças bloqueantes, adotando o princípio da estabilidade angular dos fixadores internos.

O orifício $D C P^{\circledR}$, introduzido na década de 60 , voltou a ganhar destaque após um período em que as técnicas de estabilidade absoluta deixaram de estar em evidência na literatura. 
Embora muitos estudos tenham detalhado aspectos mecânicos da utilização do orifício $D C P^{\circledR}$, descrevendo os limites de angulação em 25 graus no plano longitudinal e sete graus no plano axial, desconhecemos estudos que apontem para os efeitos que essa inclinação em relação à inserção ortogonal do parafuso excêntrico possam causar sobre a magnitude da compressão $\operatorname{produzida}^{(2-3)}$.

Esse questionamento torna-se relevante em relação à direção da inclinação. A angulação do parafuso no mesmo sentido da rampa de deslizamento do orifício $D C P^{\circledR}$ pode produzir uma compressão axial diferente daquela obtida por um parafuso inclinado na direção oposta à da inclinação da rampa.

O objetivo desta pesquisa foi analisar os efeitos mecânicos causados pela variação da inclinação do parafuso excêntrico, em relação aos planos longitudinal e axial da placa $D C P^{\circledR}$ não pré-tensionada, sobre a compressão axial interfragmentária.

\section{MÉTODOS}

A pesquisa foi realizada no Laboratório de Bioengenharia da Faculdade de Medicina de Ribeirão Preto da Universidade de São Paulo - USP. Utilizamos cilindros de poliuretano medindo $2,5 \mathrm{~cm}$ de diâmetro e $10 \mathrm{~cm}$ de comprimento, com superfície lisa, sem irregularidades e com orifício interno simulando o canal medular do osso (Synbone ${ }^{\circledR}$, Suíça). Selecionamos placas $D C P^{\circledR}$ estreitas $(4,5 \mathrm{~mm})$ e parafusos corticais de $4,5 \mathrm{~mm}$, autoperfurantes, confeccionados em aço inoxidável ASTM F138 segundo as normas NBR-ISO-5832-1/ISO5832-1, contendo sete orifícios (Synthes ${ }^{\circledR}$, Brasil). Os parafusos foram inseridos nos orifícios da placa com chave sextavada adaptada a um torquímetro calibrado. Adotamos o torque de $4 \mathrm{Nm}$ como parâmetro final de aperto para cada ensaio mecânico. Perfurações precisas em relação aos orifícios da placa foram confeccionadas junto à Oficina de Precisão Mecânica do Campus da USP de Ribeirão Preto. Foram utilizados guias de perfuração excêntrica contemplando oito diferentes situações de inclinação. Em relação ao plano longitudinal, foram quatro medidas angulares distintas: $0^{\circ}, 10^{\circ}, 20^{\circ}$ e $25^{\circ}$. Nesse plano foram consideradas não apenas as referidas inclinações como também

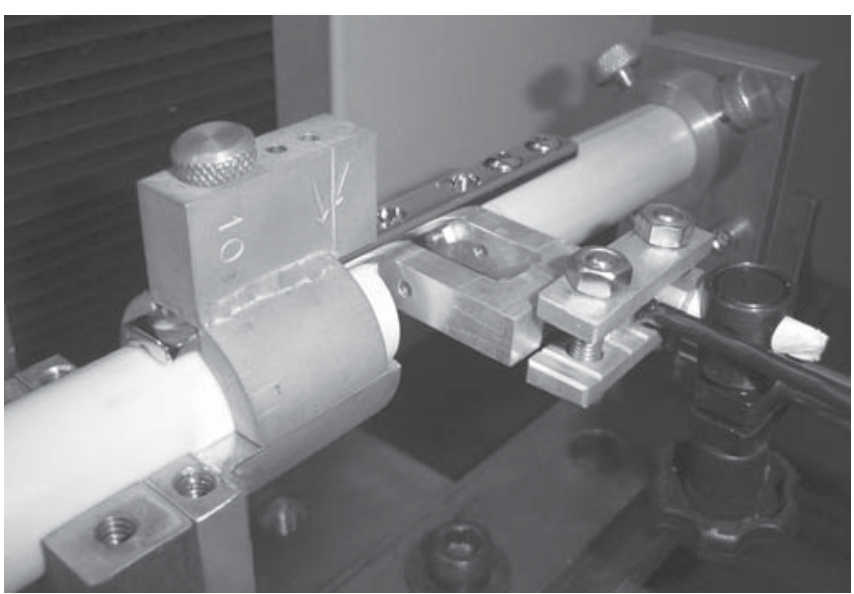

Figura 1 - Ilustração do sistema de suporte na máquina universal de ensaios

a direção da mesma em relação à rampa do orifício $D C P^{\circledR}$. Dessa maneira, obtivemos perfurações com inclinações que acompanhavam a direção da rampa, assim como perfurações com inclinações na direção contrária à mesma. Um guia de perfuração com $5^{\circ}$ de inclinação em relação ao plano transversal também foi confeccionado para o experimento, totalizando oito guias distintos.

Elaboramos guias em latão semicircular em associação a um dispositivo de suporte para o corpo de prova de poliuretano. Um pino foi adaptado ao guia em latão semicircular, para efetuar o travamento no orifício adjacente da placa $D C P^{\circledR}$. Esse dispositivo assegurava que a perfuração estava sendo realizada com excentricidade no orifício-alvo, assim como a estabilidade necessária ao guia para que a perfuração ocorresse de maneira reprodutível em todos os espécimes. Confeccionamos junto à Oficina de Precisão Mecânica uma célula de carga para a obtenção das medidas de compressão axial. Esse dispositivo foi interposto no intervalo entre dois cilindros de poliuretano, naquele que seria o virtual foco de fratura ou de osteotomia (figura 1). Constituímos oito grupos classificados quanto à inclinação do parafuso em relação aos planos longitudinal e transversal da placa. Realizamos as medidas de compressão axial com célula de carga em duas posições distintas do virtual foco de fratura ou osteotomia. As posições consideradas foram as da cortical subjacente à placa e cortical oposta à placa. Os 
dados obtidos pela pesquisa foram a força máxima (Fmax) ou pico máximo, que é caracterizada pelo pico de força registrado ao inserirmos o parafuso na posição excêntrica com o torque de $4 \mathrm{Nm}$, assim como, pela queda abrupta da magnitude até se estabilizar em um platô residual, por nós denominado força máxima efetiva (Fmax ef) ou força resultante.

\section{Análise estatística}

O método de análise de variância (ANOVA) para medidas repetidas foi utilizado para as variáveis força máxima (Fmax) e força máxima efetiva (Fmax ef). Utilizamos o método de Dunnet para as comparações post hoc. Considerou-se grupo controle aquele com ângulo de inclinação de $0^{\circ}$ em relação ao orifício da placa, com os valores de referência da posição subjacente à placa. A normalidade foi verificada por meio do teste de Shapiro-Wilks, recomendado para amostras com $\mathrm{n} \leq 20$.

O nível de significância $\mathrm{p} \leq 0,05$ foi adotado e os testes estatísticos realizados com o programa estatístico SPSS ${ }^{\circledR}$ versão 10.0 (Chicago, EUA).

\section{RESULTADOS}

\section{Comparação intercortical com inclinação $0^{\circ}$}

As medidas evidenciaram que, ao inserirmos o parafuso de maneira ortogonal na posição excêntrica do orifício $D C P^{\circledR}$, houve diferenças significativas pelo teste post hoc de Dunnet em relação à compressão axial nas diferentes posições estudadas: subjacente $>$ oposta (p $<0,001$ ) (gráfico $1 \mathrm{~A}$ e B). Na cortical oposta à placa observamos valores em média $61,2 \%$ inferiores aos registrados na cortical subjacente ao implante. Essa tendência de superioridade de medidas da Fmax na cortical subjacente à placa foi mantida em todos os demais ensaios, independente da inclinação do parafuso (tabela 1).

Força máxima (Fmax) ou pico máximo: na cortical subjacente à placa: evidenciamos que para as medidas de compressão, a inclinação de $0^{\circ}$ foi equivalente estatisticamente a $10^{\circ}$ e $20^{\circ}$ de inclinação longitudinal. Entre os outros grupos houve diferença significativa quando comparados com o grupo de controle de $0^{\circ}$

\section{A Inclinação 0 grau subjacente}
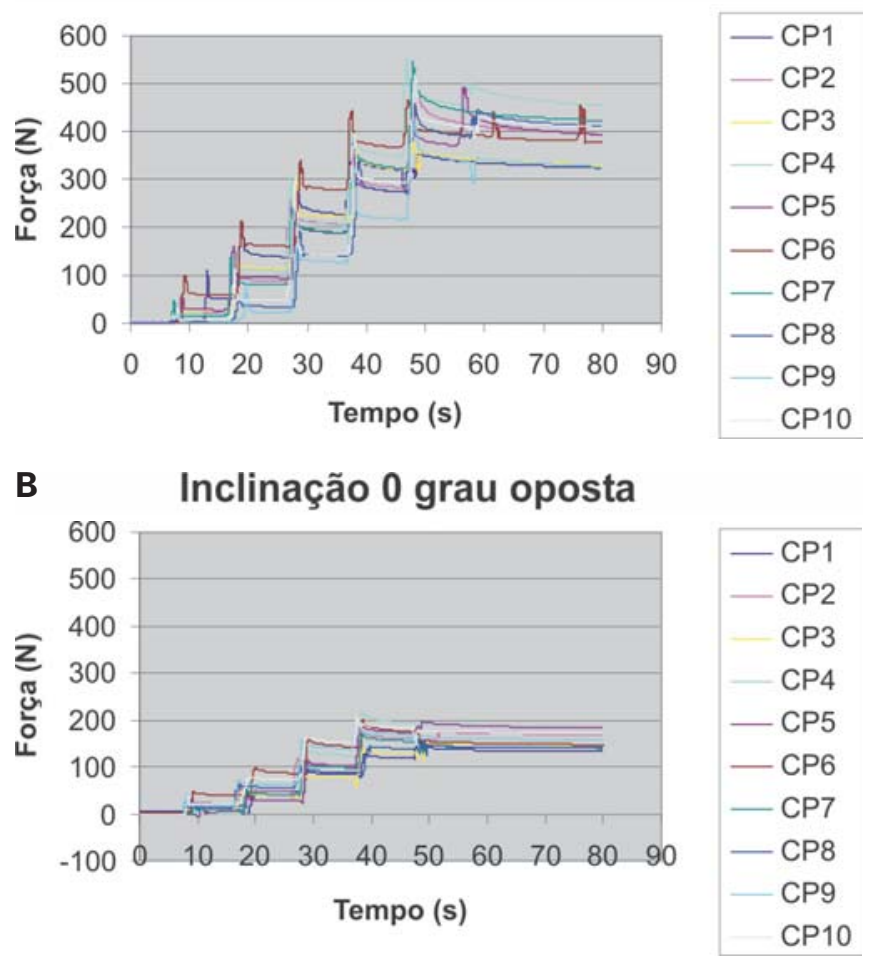

Gráfico 1 - Demonstração de valores médios da força máxima de compressão axial posições do virtual foco de fratura. A) Cortical subjacente à placa; B) Cortical oposta à placa.

TABELA 1

Valores médios de força máxima de compressão nas duas posições distintas do foco virtual de fratura

\begin{tabular}{lcc}
\hline \multicolumn{1}{c}{ Posição } & Média & Desvio-padrão \\
\hline Subjacente & 467,59 & 66,32 \\
Oposta & 181,36 & 22,11 \\
\hline
\end{tabular}

pelo teste post hoc de Dunnet. As diferenças comparativas entre os grupos estão detalhadas a seguir:

$0^{\circ}>25^{\circ}(\mathrm{p}=0,04) ; 0^{\circ}>10^{\circ} \mathrm{i}(\mathrm{p}<0,001) ; 0>20^{\circ} \mathrm{i}(\mathrm{p}$ $<0,001) ; 0^{\circ}>25^{\circ} \mathrm{i}(\mathrm{p}<0,001) ; 0^{\circ}>5^{\circ} \mathrm{t}(\mathrm{p}=0,004)$ (tabela 2).

Força máxima efetiva (Fmax ef) ou força resultante (platô): na cortical subjacente à placa, o grupo $0^{\circ}$ foi equivalente estatisticamente ao grupo $20^{\circ}$. Entre os outros grupos houve diferença significativa quando comparados com o grupo de controle de $0^{\circ}$ pelo teste post hoc de Dunnet. As diferenças estão ilustradas a seguir: 
$0>10(\mathrm{p}=0,02) ; 0>25(\mathrm{p}=0,001) ; 0>10 \mathrm{i}(\mathrm{p}<$ 0,001); $0>20 \mathrm{i}(\mathrm{p}<0,001) ; 0>25 \mathrm{i}(\mathrm{p}<0,001) ; 0>5 \mathrm{t}$ $(\mathrm{p}=0,003)($ tabela 3$)$.

TABELA 2

Valores médios e desvios-padrão da força máxima de compressão axial obtidos na cortical subjacente à placa nos diferentes grupos estudados

\begin{tabular}{ccc}
\hline Ângulo (graus) & Média (N/ m) & Desvio-padrão \\
\hline 0 & 467,59 & 66,32 \\
10 & 469,29 & 36,87 \\
20 & 499,54 & 37,41 \\
25 & 406,16 & 49,67 \\
$10 \mathrm{i}$ & 349,80 & 65,43 \\
$20 \mathrm{i}$ & 203,64 & 50,29 \\
$25 \mathrm{i}$ & 146,35 & 43,09 \\
$5 \mathrm{t}$ & 370,00 & 54,71 \\
\hline
\end{tabular}

TABELA 3

Valores médios e desvios-padrão da força máxima efetiva obtidos na cortical subjacente à placa nos diferentes grupos estudados

\begin{tabular}{ccc}
\hline Ângulo (graus) & Média (N/ m) & Desvio-padrão \\
\hline 0 & 386,73 & 44,96 \\
10 & 346,79 & 20,57 \\
20 & 376,38 & 52,07 \\
25 & 282,07 & 52,16 \\
$10 \mathrm{i}$ & 288,92 & 54,25 \\
$20 \mathrm{i}$ & 165,56 & 43,56 \\
$25 \mathrm{i}$ & 113,31 & 33,00 \\
$5 \mathrm{t}$ & 306,91 & 44,29 \\
\hline
\end{tabular}

\section{DISCUSSÃO}

Uma das técnicas mais empregadas para se obter a estabilidade absoluta é a osteossíntese com placas de compressão axial ${ }^{(11)}$.

Embora nos últimos anos grande ênfase tenha sido atribuída à osteossíntese com estabilidade relativa, valendo-se do princípio do tutor, mediante técnicas intramedulares ou fixadores internos, o surgimento das placas $L C P^{\circledR}$ trouxe novamente à tona a necessidade do princípio da compressão interfragmentária para casos selecionados.

Para que se obtenha a compressão axial com placas de orifício do tipo $D C P^{\circledR}$, é necessário que o parafuso seja inserido excentricamente no orifício do implan$t^{(2-3,12)}$. Trata-se de uma técnica de precisão em que o uso de guias inadequados ou o posicionamento central do parafuso não produzem compressão axial ${ }^{(2,13)}$.

Procuramos saber se o uso dos guias excêntricos sempre assegura compressão axial no foco de fratura. Mais do que isso, analisamos o efeito da inclinação dos parafusos excêntricos em relação aos planos transversal e longitudinal, documentando sua interferência com a magnitude da compressão obtida. Desconhecemos, até a presente data, estudo que se tenha proposto a fazer essa avaliação.

Optamos, na realização da pesquisa, por cilindros de poliuretano, cujas propriedades mecânicas à perfuração e cujo aspecto cilíndrico com orifício central procuram reproduzir a estrutura dos segmentos diafisários de espécimes ósseos. Embora o estudo com espécimes ósseos pudesse ter sido realizado, a dificuldade em obtêlos assim como assegurar a homogeneidade da amostra nos fez optar pelo uso de corpos de prova artificiais.

Para padronização do estudo foram realizados ensaios mecânicos para análise do módulo de elasticidade do corpo de prova em poliuretano. Obtivemos média de $675,9 \mathrm{MPa}(635,2-714,3 \mathrm{MPa})$ e desvio-padrão de 26,91MPa.

Cristofolini et al e Szivek et al, após análises biomecânicas, consideraram os espécimes ósseos de fêmur em poliuretano adequados para ensaios mecânicos, especialmente em análises comparativas, provendo elevada reprodutibilidade ${ }^{(14-15)}$. Esses trabalhos ratificaram a nossa escolha pelos espécimes homogêneos.

Como desejávamos estudar a compressão interfragmentária com placa, procuramos utilizar um implante já estudado em ensaios de compressão axial, que é a placa $D C P^{\circledR(3,16)}$.

Considerado um implante talvez ultrapassado atualmente, a placa $D C P^{\circledR}$ não foi descartada neste estudo, uma vez que placas de última geração, $L C P^{\circledR}$, possuem o desenho do orifício $D C P^{\circledR}$ como parte da sua unidade de acoplamento a parafusos ${ }^{(10,17-20)}$. Outro argumento favorável à escolha da placa $D C P^{\circledR}$ consiste no fato de que novos implantes como as placas $L C-D C P^{\circledR}$ ou $L C P^{\circledR}$ possuem superfície de contato ósseo bastante irregular, com ranhuras, o que poderia resultar em variações da área seccional transversa no sítio da fratura ${ }^{(21)}$. Nas fraturas diafisárias submetidas à redução anatômica e com- 
pressão axial com placa, a moldagem e pré-tensionamento do implante são elementos essenciais da técnica cirúrgica $^{(22)}$. A pré-tensão da placa ocorre manualmente no ato cirúrgico e está sujeita a variações angulares, de acordo com os instrumentos utilizados para realizá-la, a força do cirurgião e a região da placa onde se aplica a força. Como estas seriam variáveis difíceis de controlar no estudo, optamos pelo uso de placas retas.

O trabalho agregou a preocupação de realizarmos perfurações excêntricas nos orifícios da placa $D C P^{\circledR}$ com variações angulares preestabelecidas. Para isso, foi necessário o desenvolvimento de guias que assegurassem precisão angular e estabilidade suficiente durante a perfuração, não permitindo que a broca escorregasse. Vários protótipos foram desenvolvidos até que se chegasse ao modelo final, que envolvia um suporte capaz de abraçar o conjunto placa-cilindro de poliuretano. $\mathrm{O}$ fato de trabalharmos com guias fixos ao implante nos assegurou reprodutibilidade nas perfurações, fato que não teria sido possível com perfurações à mão livre.

Julgamos pertinente considerar as duas variáveis para avaliar a compressão axial obtida. Entretanto, era necessário padronizar os sítios de medida da compressão interfragmentária. Para tanto, tomamos dois pontos de referência.

A falta de pré-tensionamento da placa conduziu a medidas de força máxima e força máxima efetiva mais elevadas na posição correspondente à cortical subjacente à placa, quando comparada com a cortical oposta. Esses achados corroboram os da literatura existente $\mathrm{e}^{(3,13,22-24)}$. Eles nos fizeram considerar os valores obtidos na cortical subjacente como os mais significativos para interpretação de resultados.

Autores ratificam a possibilidade de se obter compressão axial inserindo parafusos inclinados em relação ao orifício da placa $D C P^{\circledR(25-27)}$. Entretanto, a factibilidade de inserção do parafuso excêntrico pode não significar que o mesmo esteja realizando compressão axial equivalente à verificada durante a inserção de um parafuso ortogonal à placa.

Quando analisamos o impacto da inclinação do parafuso sobre a compressão axial obtida, verificamos, de maneira geral, que variações angulares em relação à inserção ortogonal do parafuso no orifício $D C P^{\circledR}$ tenderam a cursar com quedas relativas de força máxima e força máxima efetiva. Essa tendência corrobora nossa hipótese de que as inclinações dos parafusos nos orifícios $D C P^{\circledR}$, embora tecnicamente possíveis, interferem com a magnitude da compressão axial resultante. A interferência na magnitude, entretanto, não demonstrou significância para a maioria dos grupos em que a inclinação do parafuso ocorreu no sentido longitudinal e na mesma direção da inclinação da rampa do orifício $D C P^{\circledR}$ (quadro 1 ).

\section{QUADRO 1}

Análise comparativa das posições subjacente e oposta no foco virtual da fratura em relação à força máxima e à força máxima efetiva

\begin{tabular}{|c|c|c|}
\hline Posições & Força máxima & $\begin{array}{c}\text { Força máxima } \\
\text { efetiva }\end{array}$ \\
\hline $\begin{array}{l}\text { Posição subjacente } \\
\text { Posição oposta }\end{array}$ & $\begin{array}{c}0^{\circ}=10^{\circ}=20^{\circ} \\
0^{\circ}=10^{\circ}=20^{\circ}=10^{\circ} \mathrm{i}\end{array}$ & $\begin{aligned} 0^{\circ} & =20^{\circ} \\
0^{\circ}=10^{\circ} & =20^{\circ}=5^{\circ} t\end{aligned}$ \\
\hline
\end{tabular}

Quando tomamos como referência a cortical subjacente à placa, onde foram registrados os maiores valores de Fmax e de Fmax ef, observamos que o uso de parafusos inclinados no plano transversal ou ainda no plano longitudinal com direção contrária à da rampa do orifício $D C P^{\circledR}$ causaram diferenças significativas para menos na magnitude da compressão axial obtida. Esse é um dado, a nosso ver inédito, para o qual não encontramos equivalente na literatura. As inclinações no plano longitudinal com direção oposta à da rampa de inclinação do orifício $D C P^{\circledR}$ foram ainda as que cursaram com as maiores quedas percentuais $(\mathrm{p}<0,05)$ de Fmax e Fmax ef na cortical subjacente à placa (quadro 2). Embora não tenhamos achado suporte na literatura para discutir estes achados, acreditamos que os mesmos possam ser explicados pelo fato de que o parafuso inserido na inclinação oposta ao da rampa de inclinação do orifício $D C P^{\circledR}$ não segue a direção do componente cilíndrico inclinado que compõe o orifício $D C P^{\circledR}$. Ao contrário, ele adentra o orifício de maneira oposta, ajustando-se à posição final sem seguir o deslizamento preconizado para a secção esférica da cabeça do parafuso. 


\section{QUADRO 2}

Percentual de alteração no valor da força máxima

e força máxima efetiva obtido na cortical subjacente ao foco virtual de fratura com inclinação contrária à da rampa de deslizamento

\begin{tabular}{|ccc|}
\hline Angulação (graus) & Força máxima (\%) & $\begin{array}{c}\text { Força máxima } \\
\text { efetiva (\%) }\end{array}$ \\
\hline $10^{\circ} \mathrm{i}$ & 25,2 & 25,3 \\
$20^{\circ} \mathrm{i}$ & 56,4 & 57,2 \\
$25^{\circ} \mathrm{i}$ & 68,7 & 70,7 \\
\hline
\end{tabular}

\section{CONCLUSÕES}

A introdução do parafuso com inclinação contrária à da rampa do orifício das placas de autocompressão diminui a força de compressão axial oferecida pelo sistema no foco de fratura.

\section{REFERÊNCIAS}

1. Danis R. Les coapteurs et les coapteurs juneles. Theorie et practique de l'ostesynthese. Paris : Masson et Cie Éditeurs; 1949. p. 95-105.

2. Muller ME, Allgower M, Schneider R. Manual of internal fixation: techniques recommended. 3a ed. Berlin: Springer; 1991.

3. Perren SM, Russemberger M, Steinemann S, Müller ME, Allgöwer M. A dynamic compression plate. Acta Orthop Scand Suppl. 1969;125:31-41.

4. Mast J, Jakob R, Ganz R. Planning and reduction techniques in fracture surgery. Berlin: Springer; 1989.

5. Leunig M, Hertel R, Siebenrock KA, Ballmer FT, Mast JW, Ganz R. The evolution of indirect reduction techniques for the treatment of fractures. Clin Orthop Relat Res. 2000;(375):7-14.

6. Kregor PJ, Stannard JP, Cole PA, Zlowodski M, Alonso JA. Prospective clinical trail of the less invasive stabilization system (LISS) for supracondylar femur fractures. Proceedings of Orthopedics Trauma Association Annual Meeting. J Orthop Trauma. 2000;14:133-4.

7. Marti A, Fankhauser C, Frenk A, Cordey J, Gasser B. Biomechanical evaluation of the less invasive stabilization system for the internal fixation of distal femur fractures. J Orthop Trauma. 2001;15(7):482-7.

8. Stoffel K, Dieter U, Stachowiaki G, Gächter A, Kuster MS. Biomechanical testing of the LCP - how can stability in locked internal fixators be controlled? Injury. 2003;34(Suppl 2):B11-9.

9. Wagner M, Frigg R. Locking Compression Plate (LCP): Ein neuer AO-Standard. OP J. 2000;16(3):238-43.

10. Uhthoff HK, Poitras P, Backman DS. Internal plate fixation of fractures: short history and recent developments. J Orthop Sci. 2006;11(2):118-26.

11. Perren SM, Russenberger M, Steinemann S, Muller ME, Allgower MA, et al. Cortical bone healing. Acta Orthop Scand Suppl. 1969;125:1-6.
12. Perren SM, Huggler A, Russemberger M, Straumann F, Müller ME, Allgöwer M. A method of measuring the change in compression applied to living cortical bone. Acta Orthop Scand Suppl. 1969;125:7-16.

13. Schatzker J. Principles of internal stable fixation (The Canadian Journal of Surgery). In: Uhthoff HK, editor. Current concepts of internal fixation of fractures. Berlin; New York: Springer Verlag; 1980. p. 180-1.

14. Cristofolini L, Viceconti M, Cappello A, Toni A. Mechanical validation of whole bone composite femur models. J Biomech. 1996;29(4):525-35. Comment in: J Biomech. 1996;29(9):1241.

15. Szivek JA, Gealer RL. Comparison of the deformation response of synthetic and cadaveric femora during simulated one-legged stance. J Appl Biomater. 1991;2(4):277-80.

16. Allgower M, Matter P, Perren SM, Ruedi T. The dynamic compression plate - DCP. Berlin: Springer-Verlag; 1973.

17. Miclau T, Martin RE. The evolution of modern plate osteosynthesis. Injury. 1997;28(Suppl 1):A3-6.

18. Frigg R. Locking Compression Plate (LCP). An ostesynthesis plate based on the Dynamic Compression Plate and the Point Contact Fixator (PC-Fix). Injury. 2001;32(Suppl 2):63-6.

19. Perren SM. Evolution of the internal fixation of long bone fractures. The scientific basis of biological internal fixation: choosing a new balance between stability and biology. J Bone Joint Surg Br. 2002;84(8):1093-110.

20. Egol KA, Kubiak EN, Fulkerson E, Kummer FJ, Koval KJ. Biomechanics of locked plates and screws. J Orthop Trauma. 2004;18(8):488-93.

21. Ellis T, Bourgeault CA, Kyle RF. Screw position affects dynamic compression plate strain in an in vitro fracture model. J Orthop Trauma. 2001;15(5):333-7.

22. Hayes WC, Grens WB, Murch SA, Nunamaker DM. Effects of plate modulus, thickness and pre-bending on the mechanics of compression plate fixation. Transactions of the 24th Annual Orthopaedic Research Society. Dallas Texas; 1978.

23. Cordey J, Florin P, Klaue K, Perren SM. Compression achieved with the dynamic compression plate: effects of the inclined sloping cylinder and inclination of the screw. In: Uhthoff HK, editor. Current concepts of internal fixation of fractures. Berlin: Springer-Verlag; 1980. p. 192-200.

24. Gotzen L, Hutter J, Hass N. The prebending of AO plates in compression osteosynthesis. In: Uhthoff HK, editor. Current concepts of internal fixation of fractures. Berlin: SpringerVerlag; 1980. p. 201-10.

25. Bertolini A. L'impiego della mia placca a compressione per la osteosintesi dei vari tipi di osteotomie intertrocanteriche di femore. La clinica ortopedica; 1966. p. 221-31.

26. Widolf G. A new compression plate for high femoral osteotomy for osteoarthritis of the hip. Preliminary report. Acta Orthop Scand. 1970;41(3):332-40.

27. Chaudhuri GK, Sengupta A, Saha AK. Rotation osteotomy of the shaft of the humerus for recurrent dislocation of the shoulder: anterior and posterior. Acta Orthop Scand. 1974; 45(2):193-8. 